\title{
Applying a Model of Communicative Influence in Education in Closed Online and Offline Courses
}

\author{
Caleb T. Carr \\ Illinois State University \\ School of Communication
}

\begin{abstract}
This research explores communicative influences on cognitive learning and educational affect in online and offline courses. A survey was conducted of students $(N=147)$ enrolled in online and offline courses within a single department during Summer 2013. Respondents were asked about their perceptions of classroom communication. Responses were subjected to structural equation modeling to predict each respondent's final course grade and educational affect. Results provide mixed support for Carr et al.’s [1] communicative influence in education model (CIEM), with strong and significant effects identified only for online courses. While hypotheses regarding the mediating effects of instructor credibility and social identification with co-learners were rejected, direct effects of course modality on these variables were identified. Findings are discussed with respect to differences in online and offline communication, online and offline courses, with implications for educators and institutions.
\end{abstract}

\section{INTRODUCTION}

Online education continues to grow as more institutions seek to take advantage of the benefits related to Internet-based classrooms and learning experiences. Offering courses online allows organizations to overcome the limitations of physical, on-campus classroom constraints [2], engage geographically distant student populations [3], and provide fiscal benefits to both the institution and students [4]. In 2012, 86.5\% of higher education institutions offered some online courses, with over half offering complete online degree programs [5]. The expansion of online course offerings has been matched by an increase in online enrollment $-32 \%$ of all higher education students (6.7 million) were enrolled in at least one online course in 2012 [5]. The rapid expansion of enrollment in online courses has been paralleled by the rise of significant research into the effects, opportunities, and challenges of online courses, both in delivery and outcome. Meta-analyses have repeatedly demonstrated the equivocality of educational outcomes between online and offline education [6-8]. Although online and offline education may be comparable with regard to learning outcomes, differences in class members' communication stemming from the mediation of online interactions suggests differences in the communicative influence exerted on learners.

This study builds upon previous work that has explored effects of communicative influence on student's cognitive learning in two ways. First, this research tests a theoretical model of communicative influence [1] in situ, extending its validity from the laboratory to practice. Second, this research assesses whether the model can be extended to offline education, or if the communicative processes it details are unique to computer-mediated coursework. Together, this work focuses on how mediation affects intrapersonal, interpersonal, and masspersonal communication in the classroom, and in turn how those forms of communication influence student learning. 


\section{A. The Communicative Influence in Education Model}

Communication plays a critical role in education, particularly within the classroom. Previous research has explored the influence of instructors' communication with students both in-class [eg., 9, 10] and outside of class [11], as well as how communication among students co-enrolled in a course impacts classroom experiences [12]. Often in these studies, communication scholars have isolated and focused on a single form of interaction within the classroom, be it instructor-student or student-student communication. Less research has examined the multiple communicative sources that may occur simultaneously in the classroom and influence students' experiences and performance. To begin to address this paucity of understanding of multiple communicative sources of influence, Carr et al. [1] proposed and tested the communicative influence in education model (CIEM) that concurrently addresses three sources of communicative influence within the classroom environment: Intrapersonal, interpersonal, and masspersonal communication.

Intrapersonal communication refers to an individual student's idiosyncratic and internalized view of a course and content. This self-talk about a course and its value has been conceptualized as educational affect, which Kearney [13] defined as, "an increasing internalization of positive attitudes toward the content of subject matter" (p. 81). Interpersonal communication refers to the one-to-one communication between a student and course instructor. Instructor credibility, or "students' attitudes toward the instructor as a source of communication" [14], has been identified as a critical construct within this interpersonal communication [15], i.e., students who believe the course instructor is competent, trustworthy, and caring communicator typically perform better academically [16, 17]. Finally, masspersonal communication has been defined as communication that has properties of both interpersonal and mass communication, typically allowing individuals to broadcast messages to a large number of interpersonal contacts yet enabling individual and personal feedback channels of communication [18]. While research has demonstrated interpersonal interactions with classmates influences students' perceptions of instructors and learning outcomes [19], Carr and colleagues [1] noted that online classrooms often allow individuals to communicate with and be influenced in depersonalized conditions; that is, interactions are guided by social rather than personal identities. In online settings, and particularly in courses conducted via social media, students may interact with pseudonymous others based on salient social identities rather than individuated, personalized classmates [20,21].

Findings by Carr et al. [1] generally supported the hypothesized structural model of communicative influence in online learning (see Figure 1). Positively-valenced classroom discussion regarding course content positively influenced perceptions of instructor credibility and social identification with colearners, so that individuals exposed to favorable statements about the class (e.g., "This lecture really helped me prepare for the quiz.") viewed the instructor as more credible and identified more strongly with their institutional identity. Instructor credibility and social identification with co-learners positively influenced an individual's educational affect-participants felt more interested and enabled in their own learning when the instructor was perceived as credible and when they strongly identified with their colearners as an aggregate, social group. And while instructor credibility positively affected learning outcomes and social identification with co-learners negatively influenced learning outcomes, educational affect was found to have no significant effect on exam performance-counter to a priori hypothesizing, no significant differences on exam scores were determined based on a participant's personal concern for the course. Holistically, the CIEM comprehensively models communicative influence in education on cognitive learning and educational affect facilitated via social media. However, the model and its initial test have several limitations not addressed in its explication and initial empirical test. Specifically, it is unclear whether similar effects could be expected in closed-environments and how well the model maps to communicative effects in offline education. The following sections attempt to evaluate and redress both oversights, presenting testable hypotheses to further our understanding of sources of communicative influence in learning. 
Figure 1.

Carr et al.'s (2013) communicative influence in education model.

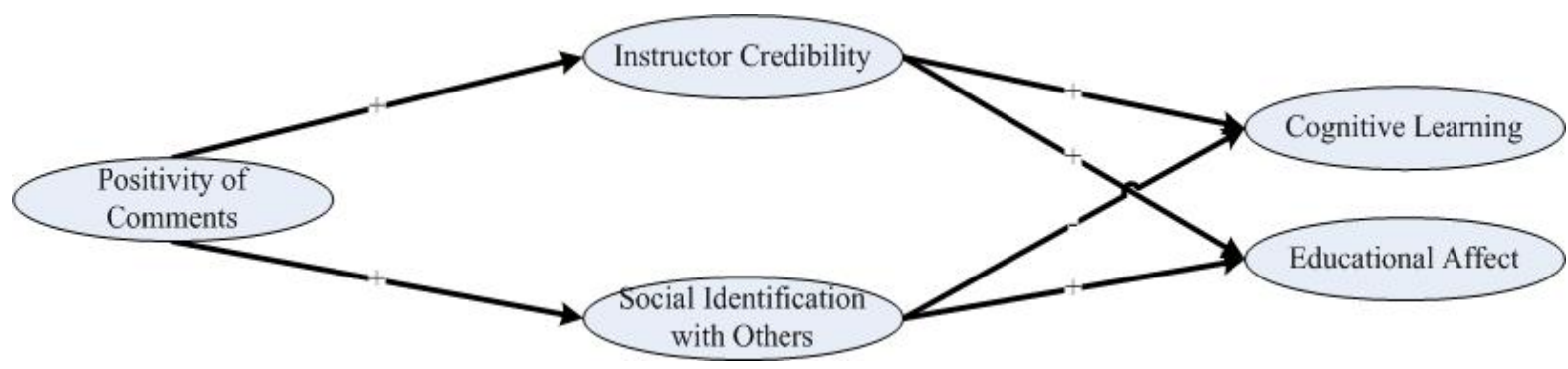

\section{B. Differences in Open and Closed Courses}

Courses, particularly those offered online, can vary widely in how accessible the course is to the public. At one end of this spectrum are open courses. Open courses are publically available for anyone to learn, engage in, and contribute [22]. Offline, individuals may find community classes open to the public or engage in course auditing whereby a college course is taken not-for-credit. Online, intuitions are beginning to utilize the power of the social, collaborative web to facilitate large-scale, publicallyavailable courses. Often labeled massive open online courses (MOOCs), web-based courses have been offered by established institutions-e.g., Stanford University offered a graduate course in computer science which drew over 58,000 globally distributed students [23]. Although open courses may allow for increased co-learner interaction, perspective-taking, and community building [24], these open fora may also allow individuals to lurk (i.e., passively observe others without contributing to classroom discussions) or to troll (i.e., maliciously poison) an online discussion [24, 25].

At the other end of the permeability spectrum are closed courses. These walled gardens of education only allow registered students and staff to access the course, limiting communicative sources to a known set of individuals: the instructor, classmates, and one's self. Traditional classrooms, in which access is limited to a fixed number of students, exemplify closed courses. Online, course management systems (CMSs; e.g., Blackboard, Desire2Learn) are almost staples of colleges both to supplement on-ground classes and deliver coursework entirely online [26]. CMSs typically facilitate closed courses, only allowing students and staff registered for and affiliated with the class access to the virtual space. While closed courses may limit diversity of perspectives and reduce participation, they can also enhance students' sense of community and foster more in-depth discussions.

The CIEM was initially developed to address communicative influence in social media. Social media are online tools relying on user contribution and interaction to develop content and value, and are closely linked to O'Reilley's [27] concept of Web 2.0 tools. Because social media emphasize interactivity and collaboration among users, coursework delivered via social media may be considered open courses, allowing anyone to access, utilize, and contribute to the course. Examples of open online classes abound, including MIT's MITx (mitx.mit.edu) and the University of West Virginia's Department of Communication Studies' programs (wvucommmooc.org). Masspersonal communication, a key tenant in the CIEM, maps nicely to the open course afforded by social media educational tools as these tools facilitate large-scale interactions that can simultaneously be interpersonal and foster mass communication.

Yet the CIEM does not specify whether its propositions would work in a closed course, which may not as readily facilitate masspersonal communication. Indeed, work into online education has typically extolled the values of interpersonal communication among classmates to interact with course content and apply classroom ideas [28, 29]. As most CMSs focus on mass communication (e.g., discussion board posts or listserves to distribute messages and replies to all enrolled learners) and interpersonal communication 
(e.g., emails and directed messages for student-student and student-student interactions), there seems little room for the facilitation of masscommunicative interaction among class members. Consequently, while Carr et al.'s [1] experiment assessed the validity of the CIEM in an open class, it did not attempt to extend or validate the model to the closed class environment which may emphasize different communicative influences. The present study sought to ameliorate this oversight by assessing the validity of the model in closed classes, to assess whether intrapersonal, interpersonal, and masspersonal communication similarly influenced students' cognitive learning. Thus:

RQ1: Does the communicative influence in education model predict cognitive learning in closed classes?

\section{Delivery Medium and Social Identification}

Another area unexplored by Carr et al's [1] model was the nuanced effect of social identification with peers on cognitive learning, guided by the social identity model of deindividuation effects [SIDE; 30]. Social identity has been defined as the, "perception of oneness with or belongingness to some human aggregate" [31], and associated with an individual's perception of himself/herself as included in a shared social category or community [32]. When individuals are depersonalized, typically via anonymity and lack of personal data disclosure, they increasingly conform to group norms and more strongly identify themselves by the salient social identity [33]. Though SIDE effects can frequently occur online given the common nature of deidentified interactions (e.g., posting to a discussion board), LeBon's [34] discussion of deindividuation driving mobs during the French Revolution also highlights SIDE effects may occur in face-to-face, collocated interactions as well. However, previous research suggests social identification in classroom settings has differing effects depending on the modality of the classroom.

Social identification with co-learners is typically considered to increase perceptions of the classroom as a community [11]. Traditional, on-ground classes have historically been considered to generate significant social identification with the classroom for students [24]; however, little research has probed the effects of social identification in the educational setting [35]. As a notable exception, Carr et al. [1] found social identification with co-learners to have a negative effect on an individual's cognitive learning when a lesson was delivered via social media tool-i.e., individuals feeling a weaker connection to the social group of co-learners performed better on an exam. Such a finding may be surprising since the SIDE model and social identification effects are often researched in the context of computer-mediated communication. However, it should be noted that SIDE was originally formulated to be applied to both online and offline processes: SIDE-guided research has only so often occurred in CMC studies because the necessary conditions to activate social identification effects are readily malleable online [cf. 36, 37]. Given the asymmetric exploration of social identification effects in education and between online and offline contexts, of initial interest is exploring the differences in social identification in online and offline courses.

In both offline and online courses, students' sense of community and social identification is primarily derived from time spent meeting and interacting with classmates [24]. In offline classes, this sense of community is developed as students spend several hours each week attending lectures, engaging in group projects, and conducting study sessions. The distributed nature of online learning facilitates equitable practices to facilitate community-building, including engaging in threaded discussions with co-learners and group projects [38, 39]. However, social processes often take longer to occur online, as the asynchronous nature of the coursework retards interaction time and textual interaction can triple the time needed for individuals to compose, read, and reply to messages as compared to face-to-face communication [40]. Most students enrolled in online programs report spending equal amounts of time engaging in both online and traditional courses [41], rather than tripling their time spent engaging in online courses to compensate for differences in education medium. The equal time spent communicating in online and offline courses should lead to greater amounts of communication and synchronous intraclass collaboration in offline classes. The larger amount of communication in offline classes would therefore be expected to produce greater perceptions of social identification with classmates as compared to online classes. Thus, it is predicted: 
H1: Social identification is significantly greater in offline classes than online classes.

Beyond affecting the magnitude of social identification, the modality of educational communication should also influence the effect of social identification on learning. Carr et al. [1] found a negative effect of social identification on cognitive learning, hypothesizing a superordinate social group identification (i.e., as Web users rather than students) may have caused individuals to focus more on the activated social identity than the social identity as a student. Consequently, strong social identification in online classes may indicate the user strongly identifies co-learners as web users rather than as students. Alternately, because the physical environment and contextual cues afforded by on-ground classes make a student social identity so salient, the in-group student identity may increase pro-learning classroom behaviors and perceptions [35]. It therefore stands to reason that social identification in offline classes leads to increased student learning, as individuals see themselves as students.

Given that online and offline courses may respectively lead to negative and positive effects on cognitive learning, it is therefore proposed the modality of course delivery moderates the influence of social identification on learning. Barron and Kenny (1986) defined a moderating variable as one that "affects the direction and/or strength of the relation between an independent or predictor variable and a dependent or criterion variable” (p. 1174). It is hypothesized the primary medium for classroom communication moderates the directionality of the relationship between social identification as a predictor variable and cognitive learning as a criterion variable. Formally:

H2a: There is a significant, negative effect of social identification on cognitive learning within online classes.

$\mathrm{H} 2 \mathrm{~b}$ : There is a significant, positive effect of social identification on cognitive learning within offline classes.

\section{Delivery Medium and Instructor Perceptions}

Finally, it may be expected the effects of students' perceptions of course instructors will differ between online and offline courses. Students' perceptions of instructors have long been acknowledged as significant antecedents of learning in offline classes. Perceptions of instructor credibility [14, 42], affect [43], and immediacy [43] have all been positively associated with classroom performance. However, one advantage offline instructors have to building positive student impressions is their ability to use multiple cues to nurture perceptions of these traits.

When collocated, individuals can use gestures, facial expressions, touch, and distance to generate positive impression formation, even in short time periods [44]. However, online communication typically restricts communicators to only verbal cues upon which perceptions may be built [45], often referred to as the cues filtered out (CFO) paradigm. Although equitable impressions can be fostered over time as individuals learn to strategically use and interpret verbal cues[46], the short, limited interactions of an online classroom may not afford the quantity or quality of interactions for individuals to form such impressions, thereby mitigating the effect. Therefore, from a CFO perspective, students enrolled in online courses should not have the cues or the time to fully develop interpersonal perceptions of the instructor, while students in offline classes should have access to abundant cues through which impressions of the instructor can be formed. Consequently, it may be predicted that the effects of instructor perceptions on a student's learning are smaller in online classes than offline classes:

H3: The medium used to deliver a course (online vs. offline) moderates the effect of instructor credibility on cognitive learning so that the effect of instructor credibility on cognitive learning is stronger in offline classes than online classes. 


\section{METHOD}

\section{A. Participants}

Eleven undergraduate courses offered in the Communication department of a Midwest university during the Summer 2013 session were contacted, with enrolled students invited to participate in the study. Participants received either course credit or extra credit for participating, commensurate with the course policies of the class from which they were recruited. Although 173 students started the study, 12 dropped the course before the end of the semester, leaving a final sample of 161 participants —an average of 14.64 participants from each course. Students enrolled in online classes were slightly oversampled $(n=107)$ as compared to students enrolled in offline classes $(n=66)$, but response rates were reflective of summer course offerings, as $60 \%$ of the departments' courses were online classes. Both online and offline courses were closed educational environments as they were only accessible to individuals registered in the course. Participants' ages ranged from 19 to $43(M=22.14, S D=3.51), 96(59.6 \%)$ respondents were female, and respondents indicated an average cumulative grade point average of $3.0(S D=.72)$ on a 4.0 scale. A simple majority of participants $(n=96,59.6 \%)$ had or intended to declare as a communication major, as measured by a binary item.

\section{B. Procedures}

At the end of the first third of each class (e.g., at the end of the second week in a 6-week course), individual course instructors forwarded an email from the researchers to students inviting them to participate in an IRB-approved online survey about their class perceptions and communication. A hyperlink was embedded in the email invitation that automatically directed participants to an online survey engine containing a consent document and the survey instrument. After completing the survey instrument, participants were thanked for their time and automatically redirected to the university's website. Surveying students at the end of the first third of the course allowed sufficient time for students to develop formative impressions and communication patterns while controlling for the relative duration of each course to have passed.

Twice while participating (first in the consent document and again at the conclusion of the survey) each participant was asked to consent to having his or her final course grade released to researchers at the conclusion of the course and after final grades had been submitted to the university's provost office. After the semester had concluded, each instructor provided course grades for participants which were then matched to survey responses. Identifying information was removed before data were analyzed.

\section{Measures}

Several items were included in the survey instrument to assess the variables of interest in this research. Positivity of comments was assessed using a novel 6-item Likert-type scale developed for this research, wherein items asked about the positivity or negativity of classroom comments targeted toward either class content, classmates, and the instructor. Items included, "Comments of others about the instructor were generally positive," "Others in the class spoke negatively about the class' content," "My classmates seemed to trust the instructor," "My classmates did not seem to view the class materials positively," "Things I heard from others in-class were positive about the content of this class," and "Others in the class spoke negatively about the instructor." Factor analysis seeking Eigenvalues greater than one revealed a one-dimension solution, and the six items demonstrated high reliability (Cronbach's $\alpha=.85$ ). The mean of the six items was computed to provide a scale measure of overall positivity of comments each participant overheard or observed from other course members regarding the course, its content, and its members.

Following earlier research into the CIEM [1], instructor credibility was assessed using McCroskey and Young's [47] 12-item teacher credibility scale (TCS). Respondents addressed instructor perceptions using a 7-point semantic differential scale with anchor points including "Unreliable|Reliable," "Awful|Nice," and "Sinful|Virtuous." Higher scores on the scale indicate the course instructor was perceived as more credible, and the scale demonstrated high reliability $(\alpha=.97)$. 
Social identification was assessed by modifying Wang's [48] 5-item social identification scale to address social identification with both online and offline others. Using 7-point Likert-type items with endpoints of 1 (strongly disagree) and 7 (strongly agree), participants indicated their agreement with statements including, "I feel involved in this class' social group," and, "This class' social group is important to me." The mean of item responses was used to assess social identification with course participants, with higher means indicating greater social identification. The scale was reliable $(\alpha=.85)$.

Educational affect was measured using a 4-item subset of McCroskey's [49] Affective Learning Scale focusing on affect toward content. Items asked respondents to respond to their perceptions of class content using 7-point semantic differential items with anchor points including, "Bad/Good," "Valuable/Worthless," "Unfair/Fair," and "Positive/Negative." The mean of item responses was used to assess affect toward course content, with higher means indicating greater educational affect. The scale demonstrated strong reliability $(\alpha=.86)$.

Finally, cognitive learning was assessed using each respondent's final course grade as a percentage of points available in the course. At the end of the scheduled semester, the course instructor provided the participant's final course grade in the class from which they were recruited and for which they provided survey responses. Though not a perfect measure of cognitive learning as it can include non-cognition based elements (e.g., participation points, extra credit), this measure of learning is common and considered valid in educational settings, and parallels previous research's [50, 51] operationalization of cognitive learning based on how an individual performs on a single exam, but on a larger scale. Participants' course grades ranged from $44.30 \%$ to $102.00 \%$, with an average of $86.12 \%(S D=9.72)$, and were leptokurtic (Kurtosis $=5.01$ ) and positively-skewed (Skewness $=-1.86$ ), but commensurate with grade distributions from previous semesters. Consequently, this method appears a valid measure of learning.

\section{ANALYSIS}

\section{A. Data Analysis}

This research predicts how communicative influences from the self, others, and the instructor can affect students' classroom learning, both online and offline. Amos 21.0 was used to conduct structural equation modeling (SEM) with maximum likelihood estimates to test hypothesized relationships. Prior to analysis, the validity of SEM assumptions was assessed by examining the data. Measurement error variance was addressed by examining data for outliers. Fourteen participants were identified as providing outlying data assessed by the Mahalanobis' $D^{2}$, and their responses were excluded from analysis, leaving $n=147$. Next, a chi-square test $(376, p<.001)$ revealed multivariate skewed and kurtosis data, and therefore nonnormality. As maximum likelihood estimation assumes normal distribution, data were normalized to meet statistical assumptions.

Finally, the hypothesized relationships were estimated using a combination of observed variable and a hybrid model [52]. As cognitive learning and educational affect were modeled as endogenous latent variables, their error terms were specified by first fixing the paths from the latent constructs of the observed variables to 1.0 . The error variance of the observed scale was then fixed to [( 1 - reliability $) \times$ scale variance] to account for the proportion of variance due to measurement error [52]. Positivity of comments, instructor credibility, social identification with contributors, and educational affect were modeled with the hybrid approach, specifying relationships between the scale items and their respective latent concepts (Table 1). As each participant's cognitive learning was assessed through a single-item measure (i.e., final course score), the observed value was used. Combining these observed variable and hybrid models allowed evaluation of the hypotheses as well as the proposed model, while accounting for measurement error and variance of the constructs.

Table 1.Standardized coefficients of latent variable indicators in structural equation model. 


\begin{tabular}{|c|c|c|c|c|}
\hline Indicator Item & $\begin{array}{l}\text { Comment } \\
\text { Positivity }\end{array}$ & $\begin{array}{c}\text { Social } \\
\text { Identification }\end{array}$ & $\begin{array}{l}\text { Instructor } \\
\text { Credibility }\end{array}$ & $\begin{array}{l}\text { Educational } \\
\text { Affect }\end{array}$ \\
\hline Positivity 1 & .510 & & & \\
\hline Positivity 2 & .587 & & & \\
\hline Positivity 3 & .665 & & & \\
\hline Positivity 4 & .725 & & & \\
\hline Positivity 5 & .754 & & & \\
\hline Positivity 6 & .757 & & & \\
\hline Identification 1 & & .668 & & \\
\hline Identification 2 & & .787 & & \\
\hline Identification 3 & & .776 & & \\
\hline Identification 4 & & .558 & & \\
\hline Identification 5 & & .741 & & \\
\hline Identification 6 & & .536 & & \\
\hline Credibility 1 & & & .867 & \\
\hline Credibility 2 & & & .803 & \\
\hline Credibility 3 & & & .919 & \\
\hline Credibility 4 & & & .843 & \\
\hline Credibility 5 & & & .887 & \\
\hline Credibility 6 & & & .889 & \\
\hline Credibility 7 & & & .592 & \\
\hline Credibility 8 & & & .961 & \\
\hline Credibility 9 & & & .894 & \\
\hline Credibility 10 & & & .871 & \\
\hline Credibility 11 & & & .907 & \\
\hline Credibility 12 & & & .882 & \\
\hline Affect 1 & & & & .752 \\
\hline Affect 2 & & & & .789 \\
\hline Affect 3 & & & & .660 \\
\hline Affect 4 & & & & .853 \\
\hline
\end{tabular}




\section{B. Hypothesis Testing}

This research sought to validate and expand Carr et al.’s [1] communicative influence in education model, replicating an experimentally-validated model of communicative influences in a social media learning environment. Central to this inquiry was exploring the CIEM as a predictor of cognitive learning in closed classes (RQ1). A structural equation model was fit to the data from closed courses offered both online and offline. The initial omnibus model, demonstrated poor model fit, $\chi^{2}(4, N=147)=18.631, p \leq$

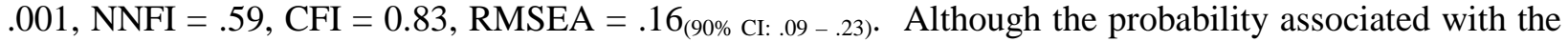
chi-squared statistic was lower than desired, the statistic was problematic to interpret given the sample sizes [52] of the study. The root mean square error of approximation demonstrated moderate fit [53]. The non-normed fit index (NNFI), comparative fit index (CFI), and root mean square error of approximation (RMSEA) are more robust statistics less influenced by sample size, and demonstrated poor fit [53] of the overall model. Given the poor fit of the model to the data, with regard to RQ1 it appears the CIEM does not generally and effectively predict cognitive learning in closed learning environments.

Given the lack of support for the CIEM for closed courses in the aggregate, additional structural equation modeling was employed to assess the validity of the model for each modality of course delivery. First, the model was fit to just the experience and responses of students enrolled in offline classes. The SEM did not serve as a good fit of the data from offline respondents, $\chi^{2}(4, n=59)=11.396, p=.022$, NNFI = .20 , CFI $=0.68$, RMSEA $=.18_{(90 \% \text { CI: } .06-.31)}$. Consequently, the model did not fit the student experience offline. Next, the model was fit to just the experience and responses of students enrolled in online classes. For online students, the SEM provided good fit to the data, $\chi^{2}(4, n=88)=5.53, p=.237$, NNFI $=.95, \mathrm{CFI}=0.98$, RMSEA $=.07_{(90 \% \mathrm{CI}: .00-.18)}$. This model provided good fit of the data [53] to the CIEM for students in closed, online courses. In other words, of the three models, only the model isolating online respondents accurately modeled the collected data.

In addition to assessing the validity of the CIEM in offline and online closed online courses, this research sought to test several hypotheses. The first hypothesis predicted social identification to be greater in offline classes than in online classes. An independent samples $t$-test was used to assess differences in reported social identification with co-learners between respondents from online and offline courses. Consistent with the hypothesized differences, respondents from offline courses reported greater social identification $(n=59, M=5.28, S D=1.00)$ than respondents from online courses $(n=88, M=4.63, S D=$ 1.12), $t(171)=3.843, p<.001$ (2-tailed), Cohen's $d=.59, r=.28$. Thus, H1 was supported.

The second hypothesis predicted a moderation effect of the delivery medium on the effect of social identification on cognitive learning. The MODPROBE analytical framework [54] was used to estimate moderation effects. Analysis revealed the medium (online or offline class delivery) did not moderate the effect of social identification on cognitive learning, $b^{*}=1.70, p=.60$. Therefore, $\mathrm{H} 2$ was rejected. However, a post hoc $t$-test revealed that, although delivery medium did not moderate the effect of social identification on cognitive learning, differences were apparent between students' levels of social identification in online $(M=4.72, S D=1.07)$ and offline $(M=5.37, S D=.86)$ classes in the expected directions, $t(145)=3.86, p<.001$. Although the mediation effect predicted by $\mathrm{H} 2$ was not supported, the post hoc analysis indicates that delivery medium may have a direct effect on social identification, an antecedent in the CIEM.

The final hypothesis predicted a moderation effect of the delivery medium on the effect of instructor credibility on cognitive learning. Although the main effect of credibility on cognitive learning was larger for offline participants $\left(b^{*}=.25, p=.04\right)$ than for online students $\left(b^{*}=.12, p=.22\right)$, no significant moderation effect was detected with the MODPROBE analysis, $b^{*}=-1.77, p=.64$. Therefore, H3 was

also rejected. Again, a post hoc t-test revealed that, although delivery medium did not moderate the effect of instructor credibility on cognitive learning, differences were apparent between students' 
perceptions of instructor credibility between delivery media, so that online instructors $(M=6.29, S D=$ $.96)$ were perceived as less credible than offline instructors $(M=6.64, S D=.60), t(145)=2.475, p=.014$. Again, though the mediation effect predicted by $\mathrm{H} 3$ was not supported, the post hoc analysis indicates delivery medium may have a direct effect on perceptions of instructor credibility, an antecedent in the CIEM.

Given the lack of support for the second and third hypotheses predicting cognitive learning, a post hoc test was conducted to assess differences in cognitive learning between online and offline courses. An independent-samples $t$-test indicated grades in online courses $(M=85.23, S D=10.97)$ were not significantly different than grades in offline courses $(M=87.62, S D=6.31), t(145)=1.509, p=.134$. An analysis of variance (ANOVA) revealed no significant difference in grades due to actual course, $F(1,137)$ $=1.279, p=.254, \eta_{\text {course }}^{2}=.077$, observed power $=.606$, suggesting differences in cognitive learning outcomes were, in part, due to differences in communicative processes of online and offline courses rather than an of medium of course delivery, specific courses, or individual instructors.

\section{DISCUSSION}

This research sought to advance Carr, Zube, Dickens, Hayter, and Barterian's [1] communicative influence in education model (CIEM). Although the CIEM demonstrated good fit in a previous study, its development and application were limited in several ways. First, the CIEM was initially tested using a controlled experimental setting that did not enable naturalistic, long-term communication amongst class participants. Second, the CIEM was developed to be applied to open education contexts-social media environments or MOOCs - and has not been applied to or validated in closed education contexts or offline classrooms. By addressing both of these limitations, the present study advances not only our understanding of the CIEM, but also of online and offline communicative influence in the process of students' education.

\section{A. Extending the CIEM}

The present research extends the scholarship surrounding the fledgling CIEM, utilizing actual classroom experiences in situ to explore and test the communicative influences predicting cognitive learning and educational affect. Even with a small sample size for SEM [cf. 53], the CIEM was well-supported for online classes, suggesting that the CIEM's communicative processes are strong and measurable in practice as well as in the laboratory. This finding provides an initial validation of the CIEM in practice and offers future research a theoretically-guided lens through which to view and understand the complex processes of online learning. However, lack of support for the model in offline courses suggests both a limitation and strength of the model: It may not extend — effectively or appropriately — to offline courses. Rather, it does provide a mechanism for understanding and exploring the complexities of classroom communication in mediated courses. As a result, based on the present findings, it seems the CIEM is best-utilized to explore the communicative processes that occur in online curricula, including counterintuitive lower levels of social identification among course members and decreased perceptions of instructor credibility.

\section{B. CIEM in Closed Courses}

Though the CIEM was supported in online classes, the present study did not find support within closed courses. The CIEM was initially developed considering the advantages of Web 2.0 tools: interactive, collaborative online learning environments facilitating open discussion among a large community of colearners. From Socrates' teachings in Greece to emergent MOOCs, these open courses have been hypothesized to lead to wider discussions among a broader group of learners [22] thereby enhancing classroom outcomes. This initial test of the CIEM in closed courses suggests communicative processes may differ in closed courses as compared to open courses. The SEM of all courses surveyed revealed a poor fit to the CIEM, indicating the communicative antecedents of cognitive learning and educational affect do not hold across online and offline courses when delivery mode is collapsed. Consequently, initial evaluation of the model, particularly in response to RQ1, confirms earlier articulations of the 
differences between open and closed courses and calls for future research to examine how classroom communication and climate may affect educational outcomes. However, this suggestion should be tempered with consideration of significant differences in the validity of the CIEM between online and offline courses.

\section{CIEM in Online Courses}

Perhaps most significant from our results surrounds support for the CIEM in online classes. This research initially sought to determine the validity of the CIEM in closed courses; however, findings indicate that though the CIEM may not be a good fit for all closed courses, it does provide a good fit for online courses. Consequently, a critical finding and distinction of this research is that the CIEM may be appropriately uniquely applied to online courses. Support for the CIEM for online courses indicates the model is an effective lens through which to explore communicative processes in online education for both open and closed courses; but moreover suggests course modality influences communicative processes more than the openness of a course.

The present findings do not reveal significant differences between students' cognitive learning based on their enrollment in an online or offline course, supporting previous research denoting the equitability of learning outcomes from online and offline courses [6-8]. However, previous research has noted the processes of online education are unique and distinct from traditional offline courses [3, 28, 55]. Post hoc analyses in this study revealed significant differences in two processes of the CIEM: Instructor credibility and social identification and instructor credibility.

The CIEM's statistical support of online courses and rejection of offline courses may likely stem from one or both of two differences between online and offline communicative factors. First, although both relationships were significant, the standardized effect of instructor credibility on educational effect was larger in online courses $\left(b^{*}=.46\right)$ than in offline courses $\left(b^{*}=.30\right)$, which alone may account for the significance of the omnibus model for respondents of online courses by accounting for additional variance. Second, consistent with previous research [24], offline students demonstrated strong social identification with their group of co-learners, more so than students in online courses. Though several scholars have noted the importance of fostering collaborative, community environments within online courses [e.g., 3, 11], the present findings suggest such processes may be limited by the technological affordances of the technology enabling online courses. This suggests a more herculean effort is needed to activate social identification processes in online courses, such as through the artificial manipulation and increased salience of a social identity for online groups [56]. Given that many online learning tools emphasize individuation (e.g., identifying and real names for student users, individualized grade books, and personalized performance metrics), future research into online communication education may explore the potential benefits of de-individuating features such as the increased use of cues to a social group and the depersonalization of class participants.

That the CIEM received significant support for closed online courses (but not for closed offline courses) suggests online learning may have common traits and experiences for learners, regardless of whether courses are open or closed. As more institutions seek to offer students online learning opportunities [57], research and models of learning need to be established and validated to understand the complex processes of computer-mediated education. Although the rejected CIEM for offline education indicates the model may not be applicable to all learning contexts, the supported CIEM for online education suggests the model may be extended to a variety of online learning contexts, and as such provides an initial framework for understanding the complex and evolving processes of online education.

\section{Future Research}

One significant limitation of the present work is the potential bias introduced by those students whose perceptions and responses are not represented in this study, either through selective non-participation or by dropping the class. Selection bias occurs when individuals choosing to participate in a study may have different perceptions or experiences than respondents who actually chose to participate, limiting the generalizability of results beyond the subset of those who were included in the study [cf. 58]. It is 
possible individuals not participating or not completing the course perceived significantly different communicative processes than those students who chose to participate. For example, students who did not see the instructor as credible may not have been motivated to participate or remain enrolled in the class, and concurrently experienced different levels of cognitive learning stemming from their perceptions of the instructor's credibility. Although there was a broad range of participants with regard to academic status, grade point average, and in-class performance in the present study, and participants were invited (rather than required) to participate in accordance with the institutional review board for human subjects research, future work that is able to capture data from all class participants may be able to ensure the significant results presented here are robust enough against nonresponse bias that they may be generalized to an even broader population.

Although this work represents an initial application of the CIEM into closed courses, it was limited to investigating individual and intergroup differences within a single institution. Particularly given the mixed support for CIEM following the strong support of the initial model, future work may seek to revisit and refine the model to account for differences in closed and open courses as well as online and offline. Such differences may readily reflect a two-by-two design that lends itself to teasing out small differences in effects and predictor-outcome relationships.

\section{CONCLUSION}

The rapid adoption of online courses and entire curricula [55, 57, 59] is outpacing the development and testing of models relating to how teachers, students, and the educational experience are adapting to the changing modality of classes. This research tested an early model of the role of communicative influences in education, seeking to address the methodological and conceptual limitations from earlier work [1]. Findings of the model in closed classrooms-learning environments whose access is limited to those formally enrolled in the course-revealed the model fits the communicative processes of online courses, but not of closed courses in general. Given the complexity of findings, the present research reinforces the need to carefully probe how diverse communicative forces uniquely influence the online learning process, and consequently how traditional pedagogical and andragogical processes may need to be adapted or reconceptualized to compensate for the radical shift to online education.

\section{REFERENCES}

1. Carr, C. T., Zube, P., Dickens, E., Hayter, C. A., \& J. A. Barterian, J. A. "Toward a model of sources of influence in online education: Cognitive learning and the effects of Web 2.0," Communication Education, 62: 61-85 (2013).

2. Parry, M. "Online, bigger classes may be better classes," Education Digest: Essential Readings Condensed for Quick Review, 76: 19-22 (2010).

3. Harasim, L. "Shift happens: Online education as a new paradigm in learning," The Internet and Higher Education, 3: 41-61 (2000).

4. Richardson, J. C. \& Swan, K. "Examining social presence in online courses in relation to students' perceived learning and satisfaction," Journal of Asynchronous Learning Networks, 7: 68-88 (2003).

5. Allen, I. E. \& Seaman, J. "Changing Course: Ten years of tracking online education in the United States," Babson Survey Research Group, Babson Park, MA2013.

6. Bernard, R. M., Abrami, P. C., Lou, Y., \& Borokhovski, E. A. Wade, L. Wozney, et al., "How does distance education compare with classroom instruction? A meta-analysis of the empirical literature," Review of Educational Research, 74: 379-439 (2004).

7. Sitzmann, T., Kraiger, K., Stewart, D., \&Wisher, R., "The comparative effectiveness of webbased and classroom instruction: A meta-analysis," Personnel Psychology, 59: 623-664 (2006).

8. Jahng, N., Krug, D., \& Zhang, Z. "Student achievement in online distance education compared to face-to-face education," European Journal of Open, Distance and E-Learning, 1: (2007).

9. Richmond, V. P., McCroskey, J. C., Kearney, P., \& Plax, T. G. "Power in the classroom VII: Linking behavior alteration techniques to cognitive learning," Communication Education, 36: 1-12 (1987). 
10. Teven, J. J. \& McCroskey, J. C. "The relationship of perceived teacher caring with student learning and teacher evaluation," Communication Education, 46: 1-9 (1997).

11. Mazer, J. P., Murphy, R. E., \& Simonds, C. J. "I'll see you on "Facebook": The effects of computer-mediated teacher self-disclosure on student motivation, affective learning, and classroom climate," Communication Education, 56: 1-17 (2007).

12. Sidelinger, R. J., Bolen, D. M., Frisby, B. N., \& McMullen, A. L. "When instructors misbehave: An examination of student-to-student connectedness as a mediator in the college classroom," Communication Education, 60: 340-361 (2011).

13. Kearney, P. "Affective learning," in Communication research measures: A sourcebook, R. B. Rubin, P. Palmgreen, and H. E. Sypher, Eds., New York, NY: Guilford Press, 1994, pp. 81-85.

14. Schrodt, P., Witt, P. L., Turman, P. D., Myers, S. A., Barton, M. H., \& Jernberg, K. A. "Instructor credibility as a mediator of instructors' prosocial communication behaviors and students' learning outcomes," Communication Education, 58: 350-371 (2009).

15. Myers, S. A. "Perceived instructor credibility and verbal aggressiveness in the college classroom," Communication Research Reports, 18: 354-364 (2001).

16. Tibbles, D., Richmond, V. P., McCroskey, J. C., \& Weber, K. "Organizational orientations in an instructional setting," Communication Education, 57: 389-407 (2008).

17. Teven, J. J. "The relationships among teacher characteristics and perceived caring," Communication Education, 50:159-169 (2001).

18. O'Sullivan, P. B. "Bridging the mass-interpersonal divide synthesis scholarship in HCR," Human Communication Research, 25: 569-588 (1999).

19. Edwards, C., Edwards, A. Qing, Q., \& Qahl, S. "The influence of computer-mediated word-ofmouth communication on student perceptions of instructors and attitudes toward learning course content," Communication Education, 55: 255-277 (2007).

20. Hiltz, S. R. "The "virtual classroom": Using computer-mediated communication for university teaching," Journal of Communication, 36: 95-104 (1986).

21. Howland, J. L., \& Moore, J. L. "Student perceptions as distance learners in Internet-based courses," Distance Education, 23: 183-195 (2002).

22. Wiid, J., Cant, M. C., \& Nell, C. "Open distance learning students' perception of the use of social media networking systems as an educational tool," International Business \& Economics Research Journal, 12: 867-882 (2013).

23. Leckart, S. (2012, March 20) The Stanford education experiment could change higher learning forever. Wired. Available: http://www.wired.com/wiredscience/2012/03/ff_aiclass/all/1

24. Arnold, N. \& Paulus, T. "Using a social networking site for experiential learning: Appropriating, lurking, modeling and community building," The Internet and Higher Education, 13: 188-196 (2010).

25. Herring, S., Job-Sluder, K., Scheckler, R., \& Barab, S. "Searching for safety online: Managing" trolling" in a feminist forum," The Information Society, 18: 371-384 (2002).

26. Harrington, C. F., Gordon, S. A., \& Schibik, T. J. "Course management system utilization and implications for practice: A national survey of department chairpersons," Online Journal of Distance Learning Administration, 7: (2004).

27. O'Reilly, T. (2005, March 3). What is Web 2.0: Design patterns and business models for the next generation of software. Available: http://oreillynet.com/pub/a/oreilly/tim/news/2005/09/30/what-isweb-20.html

28. Bach, S., Haynes, P., \& Lewis-Smith, J. Online learning and teaching in higher education. Berkshire, England: Open University Press, 2007.

29. Swan, K. "Building learning communities in online courses: The importance of interaction," Education, Communication \& Information, 3: 23-49 (2002).

30. Reicher, S. D., Spears, R., \& Postmes, T. "A social identity model of deindividuation phenomena," European Review of Social Psychology, 6: 161-198 (1995). 
31. Ashforth, B. E., \& Mael, F. "Social identity theory and the organization," Academy of Management Review, 14: 20-39 (1989).

32. Turner, J. C. "Towards a cognitive redefinition of the social group," in Social identity and intergroup relations, H. Tajfel, Ed., ed Cambridge, UK: Cambridge University Press, 1982, pp. 1540.

33. Lee, E.-J. "When and how does depersonalization increase conformity to group norms in computermediated communication?," Communication Research, 33: 423-447 (2006).

34. LeBon, G. The crowd: A study of the popular mind. London, England: Ernest Benn, 1895.

35. Edwards, C., \& Harwood, J. "Social identity in the classroom: An examination of age identification between students and instructors," Communication Education, 52: 60-65 (2003).

36. Carr, C. T. "The diametrics and modality of SIDE: A review and extension," in annual meeting of the International Communication Association, Singapore, 2010.

37. Postmes, T., Spears, R., \& Lea, M. "Breaching or building social boundaries? SIDE effects of computer-mediated communication," Communication Research, 25: 689-715 (1998).

38. Ocker, R. J., \& Yaverbaum, G. J. "Asynchronous computer-mediated communication versus face-to-face collaboration: Results on student learning, quality and satisfaction " Group Decision and Negotiation, 8: 427-440 (1999).

39. Bender, T. Discussion-based online teaching to enhance student learning: Theory, practice and assessment. Sterling, VA: Stylus, 2003.

40. Walther, J. B., \& Tidwell, L. C. "Nonverbal cues in computer-mediated communication, and the effect of chronemics on relational communication," Journal of Organizational Computing, 5: 355378 (1995).

41. Brown, A. H., \& Green, T. "Time students spend reading threaded discussions in online graduate courses requiring asynchronous participation," The International Review of Research in Open and Distance Learning, 10: 51-64 (2009).

42. Martin, M. M., Chesebro, J. L., \& Mottet, T. P. "Students' perceptions of instructors' sociocommunicative style and the influence on instructor credibility and situational motivation," Communication Research Reports, 14: 431-440 (1997).

43. Mottet, T. P., Parker-Raley, J., Beebe, S. A., \& Cunningham, C., "Instructors who resist "college lite": The neutralizing effect of instructor immediacy on students' course-workload violations and perceptions of instructor credibility and affective learning," Communication Education, 56: 145-167 (2007).

44. Ambady, N., \& Rosenthal, R. "Half a minute: Predicting teacher evaluations from thin slices of nonverbal behavior and physical attractiveness.," Journal of Personality and Social Psychology, 64: 431-441 (1993).

45. Short, J., Williams, E., \& Christie, B. The social psychology of telecommunications. London, UK: John Wiley, 1976.

46. Walther, J. B., \& Burgoon, J. K. "Relational communication in computer-mediated interaction," Human Communication Research, 19: 50-88 (1992).

47. McCroskey, J. C., \& Young, T. J. "Ethos and credibility: The construct and its measurement after three decades," Central States Speech Journal, 32: 24-34 (1981).

48. Wang, Z. "The assessment of interpersonal attraction and group identification in virtual groups," in annual meeting of the National Communication Association, Chicago, 2007.

49. McCroskey, J. C. "Assessment of affect toward communication and affect toward instruction in communication," in 1994 SCA summer conference proceedings and prepared remarks: Assessing college student competence in speech communication, S. Morreale and M. Brooks, Eds., ed Annandale, VA: Speech Communication Association, 1994.

50. Edwards, A., Edwards, C., Shaver, C., \& Oaks, M. "Computer-mediated word-of-mouth communication on RateMyProfessors.com: Expectancy effects on student cognitive and behavioral learning," Journal of Computer-Mediated Communication, 14: 368-392 (2009). 
51. Frymier, A. B., \& Houser, M. L. "The revised learning indicators scale," Communication Studies, 50: 1-12 (1999).

52. Stephenson, M. T., \& Holbert, R. L. "A Monte Carlo simulation of observable versus latent variable structural equation modeling techniques," Communication Research, 30: 332-354 (2003).

53. Byrne, B. Structural equation modeling with AMOS: Basic concepts, applications, and programming. New York, NY: Taylor and Francis, 2007.

54. Hayes, A. F., \& Matthes, J. "Computational procedures for probing interactions in OLS and logistic regression: SPSS and SAS implementations.," Behavior Research Methods, 41: 924-936 (2009).

55. Koehler, M. J., \& Mishra, P. "What happens when teachers design educational technology? The development of technological pedagogical content knowledge," Journal of Educational Computing Research, 32: 131-152 (2005).

56. Martin, J. "Virtually visual: The effects of visual technologies on online identification," in DiGRA 2005 Conference: Changing Views - Worlds in Play., 2005.

57. National Communication Association. (2012, March 19). Online courses at U.S. colleges \& universities.

Available: http://www.natcom.org/uploadedFiles/More Scholarly Resources/Data about the Discipline/PDF -C_Brief-March_2012.pdf

58. Berk, R. A. "An introduction to sample selection bias in sociological data," American Sociological Review, 48: 386-398 (1983).

59. Allen, I. E., \& Seaman, J. Going the distance: Online education in the United States, 2011, 2011, November ed. Newburyport, MA: Sloan Consortium, 2011. 